\title{
Constant mean curvature spacelike hypersurfaces in Lorentzian warped products and Calabi-Bernstein type problems
}

\author{
Juan A. Aledo ${ }^{a}$, Alfonso Romero ${ }^{b *}$ and Rafael M. Rubio ${ }^{c *}$ \\ ${ }^{a}$ Departamento de Matemáticas, E.S.I. Informática, \\ Universidad de Castilla-La Mancha, 02071 Albacete, Spain, \\ E-mail: juanangel.aledo@uclm.es \\ ${ }^{b}$ Departamento de Geometría y Topología, \\ Universidad de Granada, 18071 Granada, Spain \\ E-mail: aromero@ugr.es \\ ${ }^{c}$ Departamento de Matemáticas, Campus de Rabanales, \\ Universidad de Córdoba, 14071 Córdoba, Spain, \\ E-mail: rmrubio@uco.es
}

\begin{abstract}
In this paper we provide several uniqueness and non-existence results for complete parabolic constant mean curvature spacelike hypersurfaces in Lorentzian warped products under appropriate geometric assumptions. As a consequence of this parametric study, we obtain very general uniqueness and non-existence results for a large family of uniformly elliptic EDP's, so solving the Calabi-Bernstein problem in a wide family of spacetimes.
\end{abstract}

MSC 2010: 58J05, 53C42, 53C50

\section{Introduction}

The study of spacelike hypersurfaces with constant mean curvature (CMC) in Lorentzian manifolds has attracted the interest of a considerable group of geometers as evidenced by the amount of works that it has generated. This is due not only to its mathematic interest, but also to its relevance in General Relativity; a summary of several reasons justifying its interest can be found in [23. In particular, hypersurfaces of (non-zero) CMC are particularly suitable for studying the propagation of gravity radiation 33 . Classical papers dealing with uniqueness results on CMC 
spacelike hypersurfaces are, for instance, 9, 13 and 23. In 9, Brill and Flaherty considered a spatially closed universe, and proved several uniqueness results on CMC hypersurfaces in the large by assuming that the Ricci curvature of the spacetime satisfies that $\overline{\operatorname{Ric}}(z, z)>0$ for all timelike vector $z$. In 23, this energy condition was relaxed by Marden and Tipler to include, for instance, non-flat vacuum spacetimes. Later, Bartnik proved in 7] very general existence theorems on CMC spacelike hypersurfaces, and claimed that it would be useful to find new satisfactory uniqueness results. More recently, in [5] Alias, Romero and Sanchez proved new uniqueness results for CMC hypersurfaces in the class of spacetimes that they call closed generalized Robertson-Walker spacetimes (which includes the spatially closed Robertson-Walker spacetimes), under the Temporal Convergence Condition (TCC). Finally, in [29, Romero, Rubio and Salamanca, have provided some uniqueness results for the maximal case (zero mean curvature) in spatially parabolic generalized Robertson-Walker spacetimes, which are open models whose fiber is a parabolic Riemannian manifold.

In the paradigmatic case of CMC hypersurfaces immersed in the Lorentz-Minkowski space $\mathbb{L}^{n+1}$, $n \geq 2$, there is a great variety of results from different points of view. One of the most celebrated results is the solution to the corresponding Bernstein problem for maximal hypersurfaces, known as Calabi-Bernstein problem in this Lorentzian context, by Calabi $(n \leq 4)$ 14, and Cheng and Yau (arbitrary $n$ ) [15. As for the case of nonzero constant mean curvature, many nonlinear examples of complete spacelike hypersurfaces with nonzero constant mean curvature can be constructed (see for instance [18, 19, 34]). In [3] the spacelike hyperplanes in $\mathbb{L}^{n+1}$ are characterized as the only complete CMC spacelike hypersurfaces which are bounded between two parallel spacelike hyperplanes. On the other hand, Aiyama [2] and Xin [36] simultaneous and independently characterized spacelike hyperplanes as the only complete CMC spacelike hypersurfaces in $\mathbb{L}^{n+1}$ whose image under the Gauss map is bounded in the hyperbolic $n$-space (see also 26 for a weaker first version of this result given by Palmer). Recall that the Gauss application $N$ of a spacelike hypersurface $M$ immersed in $\mathbb{L}^{n+1}$ can be thought as an application from $M$ into the hyperbolic space $\mathbb{H}^{n} \subset \mathbb{L}^{n+1}$. Thus, the Gauss application is bounded if and only if the hyperbolic angle between $N$ and the oriented timelike axis is also bounded.

In the general case of a spacelike hypersurface in a Lorentzian manifold $\bar{M}$, the Gauss map of $M$ can be globally defined provided $\bar{M}$ is time-orientable. Although in this context it has not sense talking about bounded Gauss application, once we choose a unitary timelike vector field globally defined on $\bar{M}$ (compatible with the time-orientation), the notion of hyperbolic angle can be naturally defined (see Section 2 for the details). Thus, the assumption of bounded hyperbolic angle is the natural extension of the one used by Aiyama and Xin in their result, and actually it has been used in this context [10, 11].

In this paper we consider a wide family of Lorentzian manifolds, given by the warped product of an 1-dimensional manifold endowed with a negative definite metric and an $n$-dimensional $(n \geq 2)$ Riemannian manifold which, in general, will be taken complete and non-compact. Note that the classical spatially open Robertson-Walker cosmological models are included in that family.

In these ambient spaces there exists a distinguished unitary timelike vector field globally defined which allows to naturally define the notion of hyperbolic angle for every immersed spacelike hypersurface. Thus, one of our main aims will be to provide characterizations of complete spacelike hypersurfaces with bounded hyperbolic angle under suitable geometric hypothesis (for instance, energy-type conditions) on both the ambient space and the hypersurface. As will be pointed out, the family of warped products for which our results are applicable is very large and contains notable 
examples.

More precisely, given an $n(\geq 2)$-dimensional (connected) Riemannian manifold $\left(F, g_{F}\right)$, an open interval $I$ in $\mathbb{R}$ and a positive smooth function $f$ defined on $I$, the product manifold $I \times F$ endowed with the Lorentzian metric

$$
\bar{g}=-\pi_{I}^{*}\left(d t^{2}\right)+f\left(\pi_{I}\right)^{2} \pi_{F}^{*}\left(g_{F}\right),
$$

where $\pi_{I}$ and $\pi_{F}$ denote the projections onto $I$ and $F$, respectively, is a Lorentzian warped product in the sense of [25, cap. 7]. This kind of Lorentzian manifolds are also known as Generalized Robertson-Walker (GRW) spacetimes in the physical context [5. Along this paper we will represent this $(n+1)$-dimensional Lorentzian manifold by $\bar{M}=I \times_{f} F$. When $n=3$ and the fiber $F$ has constant sectional curvature, $\bar{M}=I \times_{f} F$ is known as a Robertson-Walker (RW) spacetime. Note that a RW spacetime obeys the cosmological principle, i.e. it is spatially homogeneous and spatially isotropic, at least locally. Thus, GRW spacetimes widely extend to RW spacetimes and include, for instance, the Lorentz-Minkowski spacetime, the Einstein-de Sitter spacetime, the Friedmann cosmological models, the static Einstein spacetime and the de Sitter spacetime. GRW spacetimes are useful to analyze if a property of a RW spacetime $M$ is stable, i.e. if it remains true for spacetimes close to $M$ in a certain topology defined on a suitable family of spacetimes [16. In fact, a deformation $s \mapsto g_{F}^{(s)}$ of the metric of $F$ provides a one parameter family of GRW spacetimes close to $M$ when $s$ approaches to 0 . Note that a conformal change of the metric of a GRW spacetime, with a conformal factor which only depends on $t$, produces a new GRW spacetime. On the other hand, a GRW spacetime is not necessarily spatially homogeneous. Recall that spatial homogeneity seems appropriate just as a rough approach to consider the universe in the large. However, this assumption could not be realistic when the universe is considered in a more accurate scale. Thus, a GRW spacetime could be a suitable spacetime to model a universe with inhomogeneous spacelike geometry [27.

When the fiber $F$ is a compact (without boundary) Riemannian manifold, the Lorentzian warped product $\bar{M}=I \times_{f} F$ is said to be spatially closed. On the other hand, if $F$ is complete and non compact, we will say that $\bar{M}$ is spatially open. In this last case, if moreover $F$ is parabolic then $\bar{M}$ is said to be spatially parabolic 29 . The open case is especially interesting since, unlike that in the closed one, it can be compatible with the inflation hypothesis and the holographic principle [6], [8]. Moreover, in a spatially open Lorentzian warped product $\bar{M}=I \times{ }_{f} F$, the boundedness of the hyperbolic angle of a spacelike hypersurface $M$ has a physical interpretation. In fact, consider the unitary normal vector field $N$ on $M$ and the unit timelike vector field $\mathcal{T}_{p}:=-\partial_{t}$ (the sign minus depends on the chosen time orientation). Along $M$ there exist two families of instantaneous observers $\mathcal{T}_{p}, p \in M$, and the normal observers $N_{p}$. The quantities

$$
\cosh \varphi(p) \quad \text { and } \quad v(p):=\left(\frac{1}{\cosh \varphi(p)}\right) N_{p}^{F},
$$

where $N_{p}^{F}$ is the projection of $N_{p}$ onto $F$ and $\varphi$ the hyperbolic angle of $M$, are respectively the energy and the velocity that $\mathcal{T}_{p}$ measures for $N_{p}$, and we have $|v|=\tanh \varphi$ on $M$, [30, pp. 45,67]. Therefore the relative speed function $|v|$ is bounded on $M$ and, hence, it does not approach to the speed of light in vacuum.

Our paper is organized as follows. In Section 2 we introduce the notation to be used for spacelike hypersurfaces in Lorentzian warped products. Section 3 is devoted to revise some results regarding the parabolicity of Riemannian manifolds, paying special attention to show under which assumption this condition can be deduced for a spacelike hypersurface of a Lorentzian warped 
product from suitable assumptions of the ambient space. In Section 4 we provide an inequality (see Lemma 21) involving the hyperbolic angle of a CMC spacelike hypersurface immersed in a Lorentzian warping product satisfying the TCC. This inequality will be the key for obtaining our results. In Section 5 we present several uniqueness and non-existence results for complete parabolic CMC spacelike hypersurfaces in Lorentzian warped products under appropriate assumptions. In particular, we widely generalized the results of Aiyama-Xin commented above. Finally, in Section 66 we apply our parametric results to the study of several Calabi-Bernstein type problems in this context. Observe that, unlike that in the case of entire graph into a Riemannian product space, an entire spacelike graph in a Lorentzian (or warped Lorentzian) product is no necessarily complete, in the sense that the induced Riemannian metric is not necessarily complete on the graph. As a non direct application of the parametric case, we obtain very general uniqueness and non-existence results for a wide family of uniformly ellyptic EDP's (see Equation (E.1)+(E.2)).

\section{Preliminaries}

Let $\left(F, g_{F}\right)$ be an $n(\geq 2)$-dimensional (connected) Riemannian manifold, $I$ an open interval in $\mathbb{R}$ and $f$ a positive smooth function defined on $I$. Then, the product manifold $I \times F$ endowed with the Lorentzian metric

$$
\bar{g}=-\pi_{I}^{*}\left(d t^{2}\right)+f\left(\pi_{I}\right)^{2} \pi_{F}^{*}\left(g_{F}\right),
$$

where $\pi_{I}$ and $\pi_{F}$ denote the projections onto $I$ and $F$, respectively, is called a Lorentzian warped product with fiber $\left(F, g_{F}\right)$, base $\left(I,-d t^{2}\right)$ and warping function $f$. Along this paper we will represent this $(n+1)$-dimensional Lorentzian manifold by $\bar{M}=I \times_{f} F$.

In any Lorentzian warped product $\bar{M}=I \times_{f} F$, the coordinate vector field $\partial_{t}:=\partial / \partial t$ is (unitary) timelike, and hence $\bar{M}$ is time-orientable. Thus, the timelike vector field $K:=f\left(\pi_{I}\right) \partial_{t}$ is also timelike. Moreover, from the relationship between the Levi-Civita connections of $\bar{M}$ and those of the base and the fiber [25, Cor. 7.35], it follows that

$$
\bar{\nabla}_{X} K=f^{\prime}\left(\pi_{I}\right) X
$$

for any $X \in \mathfrak{X}(\bar{M})$, where $\bar{\nabla}$ is the Levi-Civita connection of the Lorentzian metric (1).

Given an $n$-dimensional manifold $M$, an immersion $\psi: M \rightarrow \bar{M}$ is said to be spacelike if the Lorentzian metric (11) induces, via $\psi$, a Riemannian metric $g_{M}$ on $M$. In this case, $M$ is called a spacelike hypersurface. We will denote by $\tau=\pi_{I} \circ \psi$ the restriction of $\pi_{I}$ along $\psi$.

The time-orientation of $\bar{M}$ allows to take, for each spacelike hypersurface $M$ in $\bar{M}$, a unique unitary timelike vector field $N \in \mathfrak{X}^{\perp}(M)$ globally defined on $M$ with the same time-orientation as $-\partial_{t}$, i.e., such that $\bar{g}(N, K) \geq f(\tau):=f \circ \tau>0$ and $\bar{g}(N, K)=f(\tau)$ at a point $p \in M$ if and only if $N=-\partial_{t}$ at $p$. We will denote by $A$ the shape operator associated to $N$. Then the mean curvature function associated to $N$ is given by $H:=-(1 / n)$ trace $(A)$. As is well-known, the mean curvature is constant if and only if the spacelike hypersurface is, locally, a critical point of the $n$-dimensional area functional for compactly supported normal variations, under certain constraint of the volume. When the mean curvature vanishes identically, the spacelike hypersurface is called a maximal hypersurface. 
For a spacelike hypersurface $\psi: M \rightarrow \bar{M}$ with Gauss map $N$, the hyperbolic angle $\varphi$, at any point of $M$, between the unit timelike vectors $N$ and $-\partial_{t}$, is given by $\bar{g}\left(N, \partial_{t}\right)=\cosh \varphi$. By simplicity, throughout this paper we will refer to $\varphi$ as the hyperbolic angle function on $M$.

In any Lorentzian warped product $\bar{M}=I \times{ }_{f} F$ there is a remarkable family of spacelike hypersurfaces, namely its spacelike slices $\left\{t_{0}\right\} \times F, t_{0} \in I$. It can be easily seen that a spacelike hypersurface in $\bar{M}$ is a (piece of) spacelike slice if and only if the function $\tau$ is constant. Furthermore, a spacelike hypersurface in $\bar{M}$ is a (piece of) spacelike slice if and only if the hyperbolic angle $\varphi$ vanishes idenentically. The shape operator of the spacelike slice $\tau=t_{0}$ is given by $A=f^{\prime}\left(t_{0}\right) / f\left(t_{0}\right) I$, where $I$ denotes the identity transformation, and therefore its (constant) mean curvature is $H=-f^{\prime}\left(t_{0}\right) / f\left(t_{0}\right)$. Thus, a spacelike slice is maximal if and only if $f^{\prime}\left(t_{0}\right)=0$ (and hence, totally geodesic).

We will say that the spacelike hypersurface is contained in a slab, if it is contained between two slices. Analogously, we will say that the hypersurface is contained in an open slab if it is contained in the unbounded region determined for a slice, i.e. if there exists $t_{0} \in I$ such that $\tau>t_{0}$ (resp. $\left.\tau<t_{0}\right)$ on $M$.

\section{Parabolic Riemannian manifolds}

Because of its appearance in so many applications (Laplace equation, Helmholtz equation, etc., see for instance [32]) one of the central problems in mathematics is to understand the equation $L u=f$ on a Riemannian manifold, where $L u=\Delta u+a u$ denotes the Schrodinger operator and $a, f$ are two given function. More generally, the family of equations $\Delta u+a h(u)=f$ naturally arises in geometry in several contexts. For instance, given $(M, g)$ a 2-Riemannian manifold and $g_{1}=e^{2 u} g$ a metric pointwise conformal to $g$, if $K$ and $K_{1}$ denote the Gaussian curvatures for $g$ and $g_{1}$, respectively, then $\Delta u+K_{1} e^{2 u}=K$ where $\Delta$ is the Laplacian operator for the metric $g$.

Although this kind of equations is fairly well studied on a compact manifold without boundary, very little is known in the complete but non compact case. A non-compact Riemannian manifold is said to be parabolic if it does not admit non-constant positive superharmonic functions (see [21, for instance). Hence, the rich geometric analysis of compact Riemannian manifolds is preserved, in some sense, for complete parabolic manifolds. Moreover, the parabolicity is part of the more general problem of understanding the lack of uniqueness of solutions to the aforementioned equations.

In the two dimensional case, this notion is very close to the classical parabolicity for Riemann surfaces. Moreover, it is strongly related to the behavior of the Gaussian curvature $K$ of the surface, since every complete Riemannian surface with $K \geq 0$ is parabolic (see [1]).

Recall also that if $S$ is a complete surface and there exists a point $p_{0} \in S$ and a positive constant $r_{0}$ such that $K(p) \geq \frac{-1}{r^{2}(p) \log r(p)}$ for all $p \in S$ such that $r(p)=\operatorname{dist}\left(p_{0}, p\right) \geq r_{0}$, then $S$ is parabolic 17. On the other hand, if the negative part of $K$ is integrable on a complete surface $S$, then $S$ is parabolic $[22$.

For higher dimensions, parabolicity of Riemannian manifolds is quite different and, in particular, it has not a so direct relation with the sectional curvature of the manifold. In fact, the Euclidean space $\mathbb{R}^{n}$ is parabolic if and only if $n \leq 2$. On the other hand, if $\left(M_{1}, g_{1}\right)$ is a compact Riemannian manifold and $\left(M_{2}, g_{2}\right)$ is a parabolic Riemannian manifold, then $M_{1} \times M_{2}$ endowed with the 
product metric $g_{1}+g_{2}$ is parabolic, 21. This also works for certain warped products (see [21] for the details). In particular, the product of a compact Riemannian manifold and the real line $\mathbb{R}$ or the Euclidean plane $\mathbb{R}^{2}$ is always a parabolic Riemannian manifold.

Parabolicity is also closely related with the volume growth of the geodesic balls in an $n(\geq 2)$ dimensional non-compact complete Riemannian manifold. Indeed, if the volume growth of the geodesic balls is moderate, then the Riemannian manifold is parabolic (see [20] for the details).

The study of immersed Riemannian submanifolds (with the induced metric) in Lorentzian ambient spaces is important not only from a mathematical point of view, but also due to its relevant role in General Relativity. In particular, the case with codimension 1 (spacelike hypersurfaces) is crucial. Lorentzian warped products which admit a complete parabolic spacelike hypersurface have been studied in [29, where the following result is proved:

Let $M$ be a complete spacelike hypersurface in a Lorentzian warped product $\bar{M}=I \times_{f} F$, whose fiber has parabolic universal Riemannian covering. If the hyperbolic angle of $M$ is bounded and the restriction $f(\tau)$ on $M$ of the warping function $f$ satisfies:

i) $\sup f(\tau)<\infty$, and

ii) $\inf f(\tau)>0$,

then, $M$ is parabolic.

Conversely, let $\bar{M}=I \times_{f} F$ be a Lorentzian warped product such that its warping function $f$ satisfies i) and ii). If $\bar{M}$ admits a simply connected parabolic spacelike hypersurface $M$ whose hyperbolic angle is bounded, then the universal Riemannian covering of the fiber of $\bar{M}$ is parabolic.

Observe that in the particular case of Lorentzian warped products $\bar{M}=I \times{ }_{f} F$ with a simply connected complete parabolic Riemannian fiber, the assumption on the universal Riemannian covering is trivially satisfied. Therefore, every Lorentzian warped product with a simply connected complete parabolic Riemannian fiber, whose warping function is bounded from above and has nonzero infimum, satisfies the assumptions on the ambient space in the previous result. This is the case, for instance, of Lorentzian products (i.e. with warping function equal to 1) whose fiber is a simply connected complete parabolic Riemannian manifold.

Example 1 Taking the previous considerations into account, a complete spacelike hypersurface with bounded hyperbolic angle in the Lorentzian warped product $I \times_{f}\left(\mathbb{S}^{2} \times \mathbb{R}^{2}\right)$, where the warping function satisfies i) and ii), must be parabolic. Note also that on the 2-sphere $\mathbb{S}^{2}$ any Riemannian metric can be considered.

\section{A technical result}

Let $\psi: M \rightarrow \bar{M}$ be an $n$-dimensional spacelike hypersurface immersed in a Lorentzian warped product $\bar{M}=I \times_{f} F$. If we denote by

$$
\partial_{t}^{T}:=\partial_{t}+\bar{g}\left(N, \partial_{t}\right) N
$$


the tangential component of $\partial_{t}$ along $\psi$, then it is easy to check that the gradient of $\tau$ on $M$ is given by

$$
\nabla \tau=-\partial_{t}^{T}
$$

and so

$$
|\nabla \tau|^{2}=g_{M}(\nabla \tau, \nabla \tau)=\sinh ^{2} \varphi .
$$

Moreover, if we put $K^{T}=K+\bar{g}(K, N) N$ the tangential component of $K$ along $\psi$, a direct computation from (2) gives

$$
\nabla \bar{g}(K, N)=-A K^{T}
$$

where we have used (3), and also

$$
\nabla \cosh \varphi=-A \partial_{t}^{T}+\frac{f^{\prime}(\tau)}{f(\tau)} \bar{g}\left(N, \partial_{t}\right) \partial_{t}^{T} .
$$

On the other hand, if we represent by $\nabla$ the Levi-Civita connection of the metric $g_{M}$, then the Gauss and Weingarten formulas for the immersion $\psi$ are given, respectively, by

$$
\bar{\nabla}_{X} Y=\nabla_{X} Y-g_{M}(A X, Y) N
$$

and

$$
A X=-\bar{\nabla}_{X} N,
$$

where $X, Y \in \mathfrak{X}(M)$. Then, we get from (2), (6) and (17), that

$$
\nabla_{X} K^{T}=-f(\tau) \bar{g}\left(N, \partial_{t}\right) A X+f^{\prime}(\tau) X
$$

where $X \in \mathfrak{X}(M)$ and $f^{\prime}(\tau):=f^{\prime} \circ \tau$. Since also $K^{T}=f(\tau) \partial_{t}^{T}$, it follows from (3) and (8) that the Laplacian of $\tau$ on $M$ is

$$
\Delta \tau=-\frac{f^{\prime}(\tau)}{f(\tau)}\left\{n+|\nabla \tau|^{2}\right\}-n H \bar{g}\left(N, \partial_{t}\right) .
$$

Consequently

$$
\begin{aligned}
\Delta f(\tau) & =f^{\prime}(\tau) \Delta \tau+f^{\prime \prime}(\tau)|\nabla \tau|^{2} \\
& =-\frac{f^{\prime}(\tau)^{2}}{f(\tau)} n+|\nabla \tau|^{2} f(\tau)(\log f)^{\prime \prime}(\tau)-n H f^{\prime}(\tau) \cosh \varphi
\end{aligned}
$$

and so

$$
\begin{aligned}
\Delta(f(\tau) \cosh \varphi)= & \cosh \varphi \Delta f(\tau)+f(\tau) \Delta \cosh \varphi+2 g_{M}(\nabla f(\tau), \nabla \cosh \varphi) \\
= & -\frac{f^{\prime}(\tau)^{2}}{f(\tau)} n \cosh \varphi+f(\tau) \cosh \varphi \sinh ^{2} \varphi(\log f)^{\prime \prime}(\tau)-n H f^{\prime}(\tau) \cosh ^{2} \varphi \\
& +f(\tau) \Delta \cosh \varphi+2 g_{M}\left(A \partial_{t}^{T}, \partial_{t}^{T}\right)-2 \frac{f^{\prime}(\tau)^{2}}{f(\tau)} \cosh \varphi \sinh ^{2} \varphi
\end{aligned}
$$

where we have used (3), (44) and (5). 
On the other hand, if we assume that $M$ is a CMC hypersurface, we get from the Codazzi equation for $M$ that (see [5, Eq. 8])

$$
\Delta(f(\tau) \cosh \varphi)=\Delta \bar{g}(K, N)=\overline{\operatorname{Ric}}\left(K^{T}, N\right)+f^{\prime}(\tau) n H+f(\tau) \cosh \varphi \operatorname{trace}\left(A^{2}\right)
$$

where $\overline{\text { Ric }}$ stands for the Ricci tensor on $\bar{M}$. Therefore, from (11) and (12) we have

$$
\begin{aligned}
\overline{\operatorname{Ric}}\left(K^{T}, N\right)= & -f^{\prime}(\tau) n H-f(\tau) \cosh \varphi \operatorname{trace}\left(A^{2}\right) \\
& -\frac{f^{\prime}(\tau)^{2}}{f(\tau)} n \cosh \varphi+f(\tau) \cosh \varphi \sinh ^{2} \varphi(\log f)^{\prime \prime}(\tau)-n H f^{\prime}(\tau) \cosh ^{2} \varphi \\
& +f(\tau) \Delta \cosh \varphi+2 g_{M}\left(A \partial_{t}^{T}, \partial_{t}^{T}\right)-2 \frac{f^{\prime}(\tau)^{2}}{f(\tau)} \cosh \varphi \sinh ^{2} \varphi .
\end{aligned}
$$

If we put $N=N_{F}-\bar{g}\left(N, \partial_{t}^{T}\right) \partial_{t}$, where $N_{F}$ denotes the projection of $N$ on the fiber $F$, it is easy to obtain from (11) that

$$
\sinh ^{2} \varphi=f(\tau)^{2} g_{F}\left(N_{F}, N_{F}\right) .
$$

Besides, from [25, Chapter 7, Corollary 43] we know that

$$
\overline{\operatorname{Ric}}\left(\partial_{t}, \partial_{t}\right)=-n \frac{f^{\prime \prime}(\tau)}{f(\tau)}
$$

and

$$
\overline{\operatorname{Ric}}\left(N_{F}, N_{F}\right)=\operatorname{Ric}^{F}\left(N_{F}, N_{F}\right)+\sinh ^{2} \varphi\left(\frac{f^{\prime \prime}(\tau)}{f(\tau)}+(n-1) \frac{f^{\prime}(\tau)^{2}}{f(\tau)^{2}}\right)
$$

where $\operatorname{Ric}^{F}$ stands for the Ricci tensor on $F$ and we have used (14). Then, from (15) and (16) we obtain

$$
\begin{aligned}
\overline{\operatorname{Ric}}\left(K^{T}, N\right) & =f(\tau) \cosh \varphi \overline{\operatorname{Ric}}\left(N_{F}, N_{F}\right)-f \cosh \varphi \sinh ^{2} \varphi \overline{\operatorname{Ric}}\left(\partial_{t}, \partial_{t}\right) \\
& =f(\tau) \cosh \varphi \operatorname{Ric}^{F}\left(N_{F}, N_{F}\right)-(n-1) f(\tau) \cosh \varphi \sinh ^{2} \varphi(\log f)^{\prime \prime}(\tau) .
\end{aligned}
$$

Finally, from (13) and (17) we get

$$
\begin{aligned}
\Delta \cosh \varphi= & n H \frac{f^{\prime}(\tau)}{f(\tau)}\left(1+\cosh ^{2} \varphi\right)+\cosh \varphi\left(\operatorname{Ric}^{F}\left(N_{F}, N_{F}\right)-n \sinh ^{2} \varphi(\log f)^{\prime \prime}(\tau)\right) \\
& +\frac{f^{\prime}(\tau)^{2}}{f(\tau)} \cosh \varphi\left(n+2 \sinh ^{2} \varphi\right)+\cosh \varphi \operatorname{trace}\left(A^{2}\right)-2 \frac{f^{\prime}(\tau)}{f(\tau)} g_{M}\left(A \partial_{t}^{T}, \partial_{t}^{T}\right) .
\end{aligned}
$$

On the other hand, the square algebraic trace-norm of the Hessian tensor of $\tau$ is just

$$
|\operatorname{Hess}(\tau)|^{2}=\operatorname{trace}\left(H_{\tau} \circ H_{\tau}\right),
$$

where $H_{\tau}$ denotes the operator defined by $g_{M}\left(H_{\tau}(X), Y\right):=\operatorname{Hess}(X, Y)$ for all vector fields $X, Y \in$ $\mathfrak{X}(M)$ on $M$, and Hess is the Hessian operator for the metric $g_{M}$.

By taking the tangential component in (2) and using (3), we get that

$$
\begin{aligned}
|\operatorname{Hess}(\tau)|^{2}= & \frac{f^{\prime}(\tau)^{2}}{f(\tau)^{2}}\left((n-1)+\cosh ^{4} \varphi\right)+\cosh ^{2} \varphi \operatorname{trace}\left(A^{2}\right)+2 n H \frac{f^{\prime}(\tau)}{f(\tau)} \cosh \varphi \\
& -2 \frac{f^{\prime}(\tau)}{f(\tau)} \cosh \varphi g_{M}\left(A \partial_{t}^{T}, \partial_{t}^{T}\right) .
\end{aligned}
$$


Since $|\operatorname{Hess}(\tau)|^{2} \geq 0$, it is a straightforward computation to obtain, making use of (18) and (19), that

$$
\begin{aligned}
\cosh \varphi \Delta \cosh \varphi \geq & n H \frac{f^{\prime}(\tau)}{f(\tau)} \cosh \varphi \sinh ^{2} \varphi+\cosh ^{2} \varphi\left(\operatorname{Ric}^{F}\left(N_{F}, N_{F}\right)-n \sinh ^{2} \varphi(\log f)^{\prime \prime}(\tau)\right) \\
& +n \frac{f^{\prime}(\tau)^{2}}{f(\tau)^{2}} \cosh ^{2} \varphi+2 \frac{f^{\prime}(\tau)^{2}}{f(\tau)^{2}} \cosh ^{2} \varphi \sinh ^{2} \varphi-\frac{f^{\prime}(\tau)^{2}}{f(\tau)^{2}}\left((n-1)+\cosh ^{4} \varphi\right) .(20)
\end{aligned}
$$

Now, let us assume that the ambient spacetime satisfies the Timelike Convergence Condition (TCC). Recall that a Lorentzian manifold obeys the Timelike Convergence Condition if its Ricci tensor $\overline{\operatorname{Ric}}$ satisfies $\overline{\operatorname{Ric}}(z, z) \geq 0$, for all timelike vector $z$.Observe that (see [31, Section 2]), in our context, TCC is equivalent to

$$
f^{\prime \prime} \leq 0, \quad \operatorname{Ric}^{F}(X, X) \geq n\left(f f^{\prime \prime}-f^{\prime 2}\right) g_{F}(X, X)
$$

for all $X$ tangent to the fiber $F$. In particular, since

$$
(\log f)^{\prime \prime}(\tau)=\frac{f(\tau) f^{\prime \prime}(\tau)-f^{\prime}(\tau)^{2}}{f(\tau)^{2}}
$$

and using also (14), it follows that

$$
\operatorname{Ric}^{F}\left(N_{F}, N_{F}\right)-(n-1)(\log f)^{\prime \prime}(\tau) \sinh ^{2} \varphi \geq 0,
$$

which jointly with (20) yields

$$
\begin{aligned}
\cosh \varphi \Delta \cosh \varphi \geq & n H \frac{f^{\prime}(\tau)}{f(\tau)} \cosh \varphi \sinh ^{2} \varphi+n \frac{f^{\prime}(\tau)^{2}}{f(\tau)^{2}} \cosh ^{2} \varphi+3 \frac{f^{\prime}(\tau)^{2}}{f(\tau)^{2}} \cosh ^{2} \varphi \sinh ^{2} \varphi \\
& -(n-1) \frac{f^{\prime}(\tau)^{2}}{f(\tau)^{2}}-\frac{f^{\prime}(\tau)^{2}}{f(\tau)^{2}} \cosh ^{2} \varphi\left(1+\sinh ^{2} \varphi\right) \\
\geq & \sinh ^{2} \varphi\left(\frac{n}{2} H+\frac{f^{\prime}(\tau)}{f(\tau)} \cosh \varphi\right)^{2}+n \sinh ^{2} \varphi\left(\frac{f^{\prime}(\tau)^{2}}{f(\tau)^{2}}-\frac{n}{4} H^{2}\right)+\frac{f^{\prime}(\tau)^{2}}{f(\tau)^{2}} \sinh ^{4} \varphi .
\end{aligned}
$$

Summing up, we have proved the following result

Lemma 2 Let $\psi: M \rightarrow \bar{M}$ be an n-dimensional CMC spacelike hypersurface immersed in a Lorentzian warped product $\bar{M}=I \times_{f} F$ satisfying the TCC. Then

$$
\begin{aligned}
\cosh \varphi \Delta \cosh \varphi \geq & \sinh ^{2} \varphi\left(\frac{n}{2} H+\frac{f^{\prime}(\tau)}{f(\tau)} \cosh \varphi\right)^{2} \\
& +n \sinh ^{2} \varphi\left(\frac{f^{\prime}(\tau)^{2}}{f(\tau)^{2}}-\frac{n}{4} H^{2}\right)+\frac{f^{\prime}(\tau)^{2}}{f(\tau)^{2}} \sinh ^{4} \varphi .
\end{aligned}
$$

\section{$5 \quad$ Parametric type results}

Our aim is to use Lemma 2 in order to, under appropriate assumptions, deduce that the hyperbolic angle $\varphi$ vanishes identically (and so $M$ is a spacelike slice) or give non-existence results. In this 
sense, we will need to ask the hypersurface to be parabolic. As explained in Section 3 , this property follows automatically under certain natural hypothesis on the ambient space $\bar{M}=I \times{ }_{f} F$. However, for the sake of clarity, we have decided to state our results under the assumption of parabolicity of the hypersurface.

On the other hand, we will use of the following technical result (see, for instance, 28, Lemma 3.1]) which will allow us to obtain an upper bound for the integral of the squared length of $\nabla \cosh \varphi$ on a geodesic ball of $M$.

Lemma 3 Let $M$ be an $n(\geq 2)$-dimensional Riemannian manifold and consider $v \in C^{2}(M)$ satisfying $v \Delta v \geq 0$. Let $B_{R}$ be a geodesic ball of radius $R$ around $p \in M$. For any $r$ such that $0<r<R$ we have

$$
\int_{B_{r}}|\nabla v|^{2} d V \leq \frac{4 \sup _{B_{R}} v^{2}}{\mu_{r, R}}
$$

where $B_{r}$ denotes the geodesic ball of radius $r$ around $p \in M$ and $\frac{1}{\mu_{r, R}}$ is the capacity of the annulus $B_{R} \backslash \bar{B}_{r}$.

Let $\psi: M \rightarrow \bar{M}$ be an $n$-dimensional CMC spacelike immersed in a GRW-spacetime $\bar{M}=I \times{ }_{f} F$ satisfying TCC, and let us assume that the constant mean curvature of $M$ verifies that

$$
H^{2} \leq \frac{f^{\prime}(\tau)^{2}}{f(\tau)^{2}}
$$

Under these assumptions, from (22) and provided that $n \leq 4$ we get that

$$
\cosh \varphi \Delta \cosh \varphi \geq 0 \text {. }
$$

Then, as a consequence of Lemma 3 we have the following local estimation for the integral of the squared length of $\nabla \cosh \varphi$ on an arbitrary geodesic ball of $M$.

Theorem 4 Let $\bar{M}=I \times_{f} F$ be a Lorentzian warped product with dimension $n+1 \leq 5$ satisfying the TCC. Let $\psi: M \rightarrow \bar{M}$ be a CMC spacelike hypersurface whose mean curvature is such that

$$
H^{2} \leq \frac{f^{\prime}(\tau)^{2}}{f(\tau)^{2}}
$$

If $B_{R}$ is a geodesic ball of radius $r$ around $p \in M$, for any $r$ such that $0<r<R$, then the function $\cosh \varphi$ satisfies

$$
\int_{D_{r}}|\nabla \cosh \varphi|^{2} d V \leq \frac{C}{\mu_{r, R}}
$$

where $B_{r}$ is the geodesic ball of radius $r$ around $p \in M, \frac{1}{\mu_{r, R}}$ is the capacity of the annulus $A_{r, R}:=B_{R} \backslash \bar{B}_{r}$ and $C=C(p, R)>0$ is a constant.

Let us also assume that the hyperbolic angle $\varphi$ of $M$ is bounded. Then, there exists a positive constant $C$ such that $\cosh ^{2} \varphi \leq C$ on $M$. Thus, if we apply Lemma 3 to the function $v=\cosh \varphi$, we have for a geodesic ball $B_{R}$ of radius $R$ around $p \in M$, that for any $r$ such that $0<r<R$ the function $\cosh \varphi$ satisfies

$$
\int_{B_{r}}|\nabla \cosh \varphi|^{2} d V \leq \frac{4 C}{\mu_{r, R}}
$$


Now, if we also assume that $M$ is parabolic we have that

$$
\lim _{R \rightarrow \infty} \frac{1}{\mu_{r, R}}=0,
$$

that is, $|\nabla \cosh \varphi|^{2}$ vanishes identically on $M$ and $\operatorname{so} \cosh \varphi$ is constant on $M$. Observe that, if $H \neq 0$, from (23) we have that $f^{\prime}(\tau) \neq 0$. Finally, from (22) we conclude that $\varphi=0$ and so $M$ is a (piece of) spacelike slice.

Summing up, we can state the following result:

Theorem 5 Let $\bar{M}=I \times_{f} F$ be a Lorentzian warped product with dimension $n+1 \leq 5$ satisfying the TCC. Let $\psi: M \rightarrow \bar{M}$ be a complete parabolic CMC spacelike hypersurface with non zero mean curvature $H$ such that

$$
H^{2} \leq \frac{f^{\prime}(\tau)^{2}}{f(\tau)^{2}}
$$

and whose hyperbolic angle is bounded. Then $M$ must be a spacelike slice.

Recall that the (constant) mean curvature of the spacelike slice $\tau=t_{0}$ is given by $H=$ $-f^{\prime}\left(t_{0}\right) / f\left(t_{0}\right)$. Bearing this in mind, we are able to give the following non existence result as a direct consequence of Lemma 2

Theorem 6 Let $\bar{M}=I \times{ }_{f} F$ be a Lorentzian warped product with dimension $n+1>5$ satisfying the TCC. Then there is no complete parabolic CMC spacelike hyperfaces with non zero mean curvature $H$ such that

$$
H^{2} \leq \frac{4}{n} \frac{f^{\prime}(\tau)^{2}}{f(\tau)^{2}}
$$

and whose hyperbolic angle is bounded.

Theorem 7 Let $\bar{M}=I \times_{f} F$ be a Lorentzian warped product with dimension $n+1>2$ satisfying the TCC. Then, every parabolic complete maximal hypersurface whose hyperbolic angle is bounded and such that $\sup f(\tau)<\infty$, must be totally geodesic. Moreover, if $M$ is contained in an open slab, then $M$ must be a spacelike slice $\tau=t_{0}$, with $f^{\prime}\left(t_{0}\right)=0$.

Proof: Let $\psi: M \rightarrow \bar{M}$ be a complete parabolic maximal hypersurface whose hyperbolic angle is bounded and such that $\sup f(\tau)<\infty$. Since $\bar{M}$ satisfies the TCC, we get from (17) that $\overline{\operatorname{Ric}}\left(K^{T}, N\right) \geq 0$. Hence, from (12) we deduce that the positive function $f(\tau) \cosh \varphi$ is subharmonic. Thus, since $M$ is parabolic and $f(\tau) \cosh \varphi$ is bounded, it must be constant. Then, using again (12), we obtain that $\operatorname{trace}\left(A^{2}\right)=0$ and so $M$ is totally geodesic.

On the other hand, from Lemma 2 we know that $\cosh \varphi$ is constant, and so $f(\tau)$ must be also constant. Then, from (10) it follows that $f^{\prime}(\tau)$ vanishes identically on $M$. Now, using (9) we deduce that $\tau$ is harmonic and, since it is bounded from above or from below because $M$ is contained in an open slab, we conclude that $\tau$ is constant and $M$ is a spacelike slice.

For a Lorentzian product space $\bar{M}=I \times F$ (i.e. $f=1$ ), TCC reduces to

$$
\operatorname{Ric}^{F}(X, X) \geq 0
$$


for all $X$ tangent to the fiber $F$; that is, $\bar{M}$ satisfies TCC if and only if the fiber $F$ has non-negative Ricci curvature.

As a consequence of Theorem 7 we have

Corollary 8 Let $\bar{M}=I \times F$ be a Lorentzian product with dimension $n+1>2$ whose fiber has nonnegative Ricci curvature. Then, every complete parabolic maximal hypersurface whose hyperbolic angle is bounded must be totally geodesic. Moreover, if $M$ is contained in an open slab, then $M$ must be a spacelike slice $\tau=t_{0}$.

Remark 9 Observe that the boundedness assumption on $M$ cannot be remove in Theorem 7 and Corollary 8 as shows the known classical Calabi-Bernstein's theorem in the Lorentz-Minkowski space.

Note that for a Lorentzian warped product satisfying the TCC, if there exists $t_{0} \in I$ such that $f^{\prime}\left(t_{0}\right)=0$ then, since $f^{\prime \prime} \leq 0, t_{0}$ is a global maximum of $f^{\prime}$ and $\sup f(\tau)<\infty$. Taking this into account, we get as a consequence of Theorem 7 the following result:

Corollary 10 Let $\bar{M}=I \times_{f} F$ be a Lorentzian warped product with dimension $n+1>2$ satisfying the TCC. If there exists a maximal slice in $\bar{M}$, then every complete parabolic maximal hypersurface whose hyperbolic angle is bounded and which is contained in an open slab, must be a slice.

Finally, we have the following non-existence result for hypersurfaces in Lorentzian products.

Theorem 11 Let $\bar{M}=I \times F$ be a Lorentzian product with dimension $n+1>2$ whose fiber has non-negative Ricci curvature. Then there is no complete parabolic CMC spacelike hypersurface in $\bar{M}$ with bounded hyperbolic angle and constant mean curvature $H \neq 0$.

Proof: Suppose that there exists such a hypersurface $M$. Since $F$ has non-negative Ricci, we get from (12) that

$$
\Delta \cosh \varphi=\cosh \varphi \operatorname{Ric}^{F}\left(N_{F}, N_{F}\right)+\cosh \varphi \operatorname{trace}\left(A^{2}\right) \geq 0 .
$$

Thus, $\cosh \varphi$ is a positive and bounded subharmonic function on a parabolic Riemannian manifold, and so $\cosh \varphi$ is constant on $M$. But then trace $\left(A^{2}\right)=0$ and $M$ is totally geodesic, which is a contradiction.

The following Lemma is a consequence of the generalized maximum principle for Riemannian manifolds given by Omori 24] (see also Yau's paper [35]):

Let $M$ be a complete Riemannian manifold whose Ricci curvature is bounded away from $-\infty$ and let $u: M \longrightarrow \mathbb{R}$ be a smooth function on $M$.

a) If $u$ is bounded from above on $M$, then for each $\varepsilon>0$ there exists a point $p_{\varepsilon} \in M$ such that

$$
\left|\nabla u\left(p_{\varepsilon}\right)\right|<\varepsilon, \quad \Delta u\left(p_{\varepsilon}\right)<\varepsilon, \quad \sup u-\varepsilon<u\left(p_{\varepsilon}\right) \leq \sup u ;
$$


b) If $u$ is bounded from below on $M$, then for each $\varepsilon>0$ there exists a point $p_{\varepsilon} \in M$ such that

$$
\left|\nabla u\left(p_{\varepsilon}\right)\right|<\varepsilon, \quad \Delta u\left(p_{\varepsilon}\right)>-\varepsilon, \quad \inf u \leq u\left(p_{\varepsilon}\right)<\inf u+\varepsilon .
$$

Here $\nabla u$ and $\Delta u$ denote, respectively, the gradient and the Laplacian of $u$.

Lemma 12 Let $\psi: M \rightarrow \bar{M}$ be an $n$-dimensional $(n \geq 2)$ CMC complete spacelike hypersurface in a Lorentzian warped product $\bar{M}=I \times{ }_{f} F$ whose warping function satisfies $(\log f)^{\prime \prime} \leq 0$. If the Ricci curvature of $M$ is bounded from below and $M$ is contained between two slices, then

$$
H=-\frac{f^{\prime}(\tau)}{f(\tau)}
$$

Proof: Since $M$ is contained between two slices, the function $\tau$ is bounded from above and from below.

As $\tau$ is bounded from below, we know from the generalized maximum principle that for each positive integer $m$ there exists a point $p_{m} \in M$ such that

$$
-\frac{1}{m}>\Delta \tau\left(p_{m}\right)=-\frac{f^{\prime}\left(\tau\left(p_{m}\right)\right)^{2}}{f\left(\tau\left(p_{m}\right)\right)}\left\{n+\left|\nabla \tau\left(p_{m}\right)\right|^{2}\right\}-n H \bar{g}\left(N\left(p_{m}\right), \partial_{t}\left(p_{m}\right)\right)
$$

where we have used (9). Letting $m$ tend to infinity, and taking into account that $\bar{g}\left(N\left(p_{m}\right), \partial_{t}\left(p_{m}\right)\right)$ tends to 1 when $\left|\nabla \tau\left(p_{m}\right)\right|^{2}$ tends to 0 , we get

$$
H \leq-\frac{f^{\prime}(\inf \tau)}{f(\inf \tau)} .
$$

Analogously, since $\tau$ is bounded from above it follows that

$$
H \geq-\frac{f^{\prime}(\sup \tau)}{f(\sup \tau)}
$$

Finally, observe that the assumption $(\log f)^{\prime \prime} \leq 0$ means that $\frac{-f^{\prime}}{f}$ is an increasing function, and so

$$
H \leq-\frac{f^{\prime}(\inf \tau)}{f(\inf \tau)} \leq-\frac{f^{\prime}(\sup \tau)}{f(\sup \tau)} \leq H
$$

which finishes the proof.

It worths pointing out that Lemma 12 goes a bit further than [12, Corollary 5.3] where, under similar assumptions for the 2-dimensional case, a bound for $\mathrm{H}^{2}$ is provided.

Note also that in Lemma 12 the hypothesis on $f$ follows immediately if the ambient space satisfies the Timelike Convergence Condition. On the other hand, the lower bound for the Ricci curvature of the hypersurface can be deduced from suitable assumptions on the sectional curvature of the fiber, as the following Lemma shows: 
Lemma 13 Let $\psi: M \rightarrow \bar{M}$ be an $n$-dimensional $(n \geq 2)$ CMC complete spacelike hypersurface in a Lorentzian warped product $\bar{M}=I \times_{f} F$ whose fiber $F$ has non-negative sectional curvature and its warping function satisfies $(\log f)^{\prime \prime} \leq 0$. Then the Ricci curvature of $M$ is bounded from below.

Proof: Given $p \in M$, let us take a local orthonormal frame $\left\{U_{1}, \ldots, U_{n}\right\}$ around $p$. From the Gauss equation

$$
\langle R(X, Y) V, W\rangle=\langle\bar{R}(X, Y) V, W\rangle+\langle A Y, W\rangle\langle A X, V\rangle-\langle A Y, V\rangle\langle A X, W\rangle, \quad X, Y, V, W \in \mathfrak{X}(M)
$$

where $\bar{R}$ and $R$ denote the curvature tensors of $\bar{M}$ and $M$ respectively, and $A$ is the shape operator of $\psi$, we get that the Ricci curvature of $M$, $\operatorname{Ric}^{M}$ satisfies

$$
\operatorname{Ric}^{M}(Y, Y) \geq \sum_{k} \bar{g}\left(\bar{R}\left(Y, U_{k}\right) Y, U_{k}\right)-\frac{n^{2}}{4} H^{2}|Y|^{2}, \quad Y \in \mathfrak{X}(M) .
$$

Now, from [25, Proposition 7.42] we have

$$
\begin{aligned}
\sum_{k=1}^{n} \bar{g}\left(\bar{R}\left(Y, U_{k}\right) Y, U_{k}\right) \quad & =\sum_{k=1}^{n} g\left(R^{F}\left(Y^{F}, U_{k}^{F}\right) Y^{F}, U_{k}^{F}\right)+(n-1) \frac{f^{\prime 2}}{f^{2}}|Y|^{2} \\
& -(n-2)(\log f)^{\prime \prime} g(Y, \nabla \tau)^{2}-(\log f)^{\prime \prime}|\nabla \tau|^{2}|Y|^{2} \geq 0,
\end{aligned}
$$

where $R^{F}$ denotes the curvature tensor of the fiber $F$ and $Y^{F}, U_{k}^{F}$ are the projections of $Y, U_{k}$ on the fiber $F$. Therefore

$$
\operatorname{Ric}^{M}(Y, Y) \geq-\frac{n^{2}}{4} H^{2}|Y|^{2}
$$

which ends the proof.

From Theorem [5] and Lemmas 12 and 13 we get the following result:

Theorem 14 Let $\bar{M}=I \times{ }_{f} F$ be a Lorentzian warped product with dimension $n+1 \leq 5$, whose fiber has non-negative sectional curvature. Let $M$ be a complete parabolic CMC spacelike hypersurface with mean curvature $H \neq 0$ which is contained between two slices and whose hyperbolic angle is bounded. If the warping function satisfies $f^{\prime \prime}(\tau) \leq 0$ on $M$, then $M$ must be a spacelike slice.

Proof: It is enough to observe that, under these assumptions, (21) holds. The proof finishes as in Theorem 5 using also Lemmas 12 and 13 .

Remark 15 Note that the boundedness of the sectional curvature of a manifold does not assure its parabolicity. For instance, the Euclidean space is not parabolic, although it has zero constant sectional curvature. Neither, as shows [29, Counterexample 5.4], the parabolicity of the manifold assures the boundedness of the curvature.

Remark 16 Regarding the hypothesis in Theorem 14 note that, in principle, there does not exist any relation between the conditions "bounded hyperbolic angle" and "being contained in a slab" for a spacelike hypersurface in a Lorentzian warped product. In fact, every non horizontal spacelike hyperplane in the Lorentz-Minkowski space has bounded hyperbolic angle, but it is not contained in any slab. Conversely, as shows [11, Remark 5.3], there exist spacelike hypersurfaces in Lorentzian warped products which are contained in a slab, but whose hyperbolic angle is not bounded. 


\section{Calabi-Bernstein type Problems}

Let $\left(F, g_{F}\right)$ be a (non-compact) $n$-dimensional Riemannian manifold and $f: I \longrightarrow \mathbb{R}$ a positive smooth function. For each $u \in C^{\infty}(F)$ such that $u(F) \subset I$, we can consider its graph $\Sigma_{u}=$ $\{(u(p), p): p \in F\}$ in the Lorentzian warped product $\left(\bar{M}=I \times{ }_{f} F, \bar{g}\right)$. The graph inherits from $\bar{M}$ a metric, represented on $F$ by

$$
g_{u}=-d u^{2}+f(u)^{2} g_{F} .
$$

This metric is Riemannian (i.e., positive definite) if and only if $u$ satisfies $|D u|<f(u)$, everywhere on $F$, where $D u$ denotes the gradient of $u$ in $\left(F, g_{F}\right)$ and $|D u|^{2}=g_{F}(D u, D u)$. Note that $\tau(u(p), p)=u(p)$ for any $p \in F$, and so $\tau$ and $u$ may be naturally identified on $\Sigma_{u}$. When $\Sigma_{u}$ is spacelike, the unitary normal vector field on $\Sigma_{u}$ satisfying $\bar{g}\left(N, \partial_{t}\right)>0$ is

$$
N=-\frac{1}{f(u) \sqrt{f(u)^{2}-|D u|^{2}}}\left(f(u)^{2} \partial_{t}+D u\right),
$$

and the corresponding mean curvature function is

$$
H(u)=-\operatorname{div}\left(\frac{D u}{n f(u) \sqrt{f(u)^{2}-|D u|^{2}}}\right)-\frac{f^{\prime}(u)}{n \sqrt{f(u)^{2}-|D u|^{2}}}\left(n+\frac{|D u|^{2}}{f(u)^{2}}\right) .
$$

In what follows, we will apply the previous uniqueness and non existence results on CMC spacelike hypersurfaces given in Section [5 to study the entire solutions of the CMC spacelike hypersurface equation in Lorentzian warped product $\bar{M}=I \times_{f} F$ whose fiber is a parabolic Riemannian manifold. Concretely, we will determine, in several relevant cases, all the entire solutions of

$$
\begin{aligned}
& \operatorname{div}\left(\frac{D u}{f(u) \sqrt{f(u)^{2}-|D u|^{2}}}\right)=-n H-\frac{f^{\prime}(u)}{\sqrt{f(u)^{2}-|D u|^{2}}}\left(n+\frac{|D u|^{2}}{f(u)^{2}}\right), \\
& |D u|<\lambda f(u), \quad 0<\lambda<1 .
\end{aligned}
$$

Note that the constraint (E.2) can be written as

$$
\cosh \varphi<\frac{1}{\sqrt{1-\lambda^{2}}}
$$

where $\varphi$ is the hyperbolic angle of $\Sigma_{u}$. Therefore, (E.2) implies that $\Sigma_{u}$ has bounded hyperbolic angle. Moreover, this constraint means that the differential equation (E.1) is, in fact, uniformly elliptic.

Now recall that the induced metric on a closed spacelike hypersurface in a complete Lorentzian manifold could be non complete. Actually, there exist entire spacelike graphs in $\mathbb{L}^{n+1}$ which are non complete [4. However, an entire CMC spacelike graph in $\mathbb{L}^{n+1}$ must be complete [15]. Therefore, if we want to derive a non-parametric uniqueness result from a parametric one, we have to prove previously the completeness of the induced metric. Indeed, the assumption on $\lambda$ in (E.2) jointly with the fact that $f(u)$ does not approach to zero on $F$, will provide the completeness of $g_{u}$ on $F$ if $g_{F}$ is complete, as we prove in the following result. 
Lemma 17 Let $\bar{M}=I \times_{f} F$ be a Lorentzian warped product whose fiber is a (non-compact) complete Riemannian manifold. Consider a function $u \in C^{\infty}(F)$, with $\operatorname{Im}(u) \subseteq I$, such that the entire graph $\Sigma_{u}=\{(u(p), p): p \in F\} \subset \bar{M}$ endowed with the metric $g_{u}=-d u^{2}+f(u)^{2} g_{F}$ is spacelike. If the hyperbolic angle of $\Sigma_{u}$ is bounded and $\inf f(u)>0$, then the graph $\left(\Sigma_{u}, g_{\Sigma_{u}}\right.$ is complete, or equivalently the Riemannian surface $\left(F, g_{u}\right)$ is complete.

Proof: The classical Schwartz inequality gives

$$
g_{\Sigma_{u}}(\nabla \tau, v)^{2} \leq g_{\Sigma_{u}}(\nabla \tau, \nabla \tau) g_{\Sigma_{u}}(v, v), \text { for all } v \in T_{q}\left(\Sigma_{u}\right), q \in \Sigma_{u}
$$

and therefore

$$
g_{\Sigma_{u}}(v, v) \geq-g_{\Sigma_{u}}(\nabla \tau, \nabla \tau) g_{\Sigma_{u}}(v, v)+f(\tau)^{2} g_{F}\left(d \pi_{F}(v), d \pi_{F}(v)\right),
$$

which implies

$$
g_{\Sigma_{u}}(v, v) \geq \frac{f(\tau)^{2}}{\cosh ^{2} \varphi} g_{F}\left(d \pi_{F}(v), d \pi_{F}(v)\right),
$$

and $\sup (\cosh \varphi)<\infty$. If we denote by $\mathcal{L}(\alpha)$ and $\mathcal{L}_{u}(\alpha)$ the lengths of a smooth curve $\alpha$ on $F$ with respect to the metrics $g_{F}$ and $g_{u}$, respectively, it is not difficult to see that

$$
\mathcal{L}_{u}(\alpha) \geq B \inf (f(u)) \mathcal{L}_{u}(\alpha),
$$

where $B=\frac{1}{\sup (\cosh \varphi)}$. Therefore, since the Riemannian manifold $\left(F, g_{F}\right)$ is complete and $\inf (f(u))>$ 0 , we conclude that the metric $g_{u}$ is also complete.

Next, making use of the study developed in Section 5, we provide several results for bounded solutions to the Equation (E.1)+(E.2) under suitable assumptions.

Theorem 18 Let $(F, g)$ be a simply connected complete parabolic Riemannian $n$-manifold, $n \leq 4$, whose sectional curvature is non-negative. Let $f: I \longrightarrow \mathbb{R}^{+}$be a smooth function satisfying $f^{\prime \prime} \leq 0$. Then, the only bounded entire solutions to the Equation (E.1) $+(E .2)$ for $H \in \mathbb{R}^{*}$, are the constant functions $u=u_{0}$ with $H=-\frac{f^{\prime}\left(u_{0}\right)}{f\left(u_{0}\right)}$.

Proof: Let $u$ be an entire solution to (E.1)+(E.2). As we have commented above, the normal unitary vector field on the graph $\Sigma_{u}=\{(u(p), p): p \in F\}$ satisfying that $\cosh \varphi=\bar{g}\left(N, \partial_{t}\right)>0$ is given by (24), and hence the constraint (E.2) can be expressed as (25). Finally, making use of Lemma 17 and Theorem 14 the proof ends.

As a consequence of Lemma 17 and Theorem [6, we have

Theorem 19 Let $(F, g)$ be a simply connected complete parabolic Riemannian $n$-manifold, $n>4$. Let $f: I \longrightarrow \mathbb{R}^{+}$be a smooth function satisfying $f^{\prime \prime} \leq 0, \inf f>0, \sup f<\infty$ and

$$
\inf \operatorname{Ric}^{F} \geq n\left(f f^{\prime \prime}-f^{\prime 2}\right)
$$

If $H \in \mathbb{R}^{*}$ is such that

$$
H^{2} \leq \inf _{I}\left\{\frac{f^{\prime}(t)^{2}}{f(t)^{2}}\right\},
$$

then the Equation (E.1)+(E.2) has not entire solutions. 
Corollary 20 Let $(F, g)$ be a simply connected complete parabolic Riemannian $n$-manifold, $n>4$. Let $f: I \longrightarrow \mathbb{R}^{+}$be a smooth function satisfying $f^{\prime \prime} \leq 0$ and

$$
\inf \operatorname{Ric}^{F} \geq n\left(f f^{\prime \prime}-f^{\prime 2}\right)
$$

If $H \in \mathbb{R}^{*}$ is such that

$$
H^{2} \leq \inf _{I}\left\{\frac{f^{\prime}(t)^{2}}{f(t)^{2}}\right\}
$$

then the Equation (E.1)+(E.2) has not entire bounded solutions.

When $f=1$, we have

Theorem 21 Let $(F, g)$ be a simply connected complete parabolic Riemannian $n$-manifold, $n \geq 2$, whose Ricci curvature is non negative. Then there is no entire solutions to the equation

$$
\begin{gathered}
\operatorname{div}\left(\frac{D u}{\sqrt{1-|D u|^{2}}}\right)=-n H \\
|D u|<\lambda, \quad 0<\lambda<1,
\end{gathered}
$$

where $H \neq 0$.

Proof: It follows from Lemma 17 and Theorem 11.

We finish with some results under the assumption $H=0$, the second one for $f=1$ constant.

Theorem 22 Let $(F, g)$ a simply connected complete parabolic Riemannian $n$-manifold, $n \geq 2$. Let $f: I \longrightarrow \mathbb{R}^{+}$be a smooth function satisfying $f^{\prime \prime} \leq 0$, inf $f>0$ and $\sup f<\infty$. Then the only entire solutions to the equation

$$
\begin{gathered}
\operatorname{div}\left(\frac{D u}{f(u) \sqrt{f(u)^{2}-|D u|^{2}}}\right)=-\frac{f^{\prime}(u)}{\sqrt{f(u)^{2}-|D u|^{2}}}\left(n+\frac{|D u|^{2}}{f(u)^{2}}\right) \\
|D u|<\lambda f(u), \quad 0<\lambda<1,
\end{gathered}
$$

which are bounded from above or from below are the constants $u=u_{0}$, with $f^{\prime}\left(u_{0}\right)=0$.

Proof: It follows from Lemma 17 and Theorem 7.

Corollary 23 Let $(F, g)$ a simply connected complete parabolic Riemannian $n$-manifold, $n \geq 2$. Let $f: I \longrightarrow \mathbb{R}^{+}$be a smooth function satisfying $f^{\prime \prime} \leq 0$. Then the only bounded entire solutions to the equation

$$
\begin{gathered}
\operatorname{div}\left(\frac{D u}{f(u) \sqrt{f(u)^{2}-|D u|^{2}}}\right)=-\frac{f^{\prime}(u)}{\sqrt{f(u)^{2}-|D u|^{2}}}\left(n+\frac{|D u|^{2}}{f(u)^{2}}\right) \\
|D u|<\lambda f(u), \quad 0<\lambda<1,
\end{gathered}
$$

are the constants $u=u_{0}$, with $f^{\prime}\left(u_{0}\right)=0$. 
If, moreover, $f=1$, we have

Corollary 24 Let $(F, g)$ be a simply connected complete parabolic Riemannian $n$-manifold, $n \geq 2$, whose Ricci curvature is non negative. Then the only entire solutions to the equation

$$
\begin{gathered}
\operatorname{div}\left(\frac{D u}{\sqrt{1-|D u|^{2}}}\right)=0 \\
|D u|<\lambda, \quad 0<\lambda<1,
\end{gathered}
$$

which are bounded from above or below are the constants.

Proof: It follows from Lemma 17 and Corollary 8

Example 25 Consider the Riemannian product $\mathbb{S}^{2} \times \mathbb{R}^{2}$ of the sphere with its usual metric and the Euclidean plane. Then the only entire solutions which are bounded from above or below to the equation

$$
\begin{gathered}
\operatorname{div}\left(\frac{D u}{\sqrt{1-|D u|^{2}}}\right)=-4 H \\
|D u|<\lambda, \quad 0<\lambda<1,
\end{gathered}
$$

for $H=0$, are the constants.

Moreover, if $H \in \mathbb{R}^{*}$, then there is no entire solutions.

\section{Acknowledgments}

The first author is partially supported by the Spanish MICINN Grant with FEDER funds MTM201019821. The second and third authors are partially supported by the Spanish MICINN Grant with FEDER funds MTM2010-18099. The third author is also partially supported by the Junta de Andalucía Regional Grant with FEDER funds P09-FQM-4496.

\section{References}

[1] L. Ahlfors, Sur le type d'une surface de Riemann, C.R. Acad. Sc. Paris 201 (1935), 30-32.

[2] R. Aiyama, On the Gauss map of complete space-like hypersurfaces of constant mean curvature in Minkowski space, Tsukuba J. Math. 16 (1992), 353-361.

[3] J.A. Aledo and L.J. Alías, On the curvatures of bounded complete spacelike hypersurfaces in the Lorentz-Minkowski space, Manuscripta Math. 101 (2000), 401-413.

[4] L.J. Alías and P. Mira, On the Calabi-Bernstein theorem for maximal hypersurfaces in the Lorentz-Minkowski space, Publicaciones de la RSME 4 (2003), 23-55. 
[5] L.J. Alías, A. Romero and M. Sánchez, Uniqueness of complete spacelike hypersurfaces of constant mean curvature in Generalized Robertson-Walker spacetimes, Gen. Relativity Gravitation 27 (1995), 71-84.

[6] D. Black and S-J Rey, Cosmic Holography, Classical Quantum Gravity 17 (2000), L83-L89.

[7] R. Bartnik, Existence of maximal surfaces in asymptotically flat spacetimes, Commun. Math. Phys. 94 (1984), 155-175.

[8] R. Bousso, The holographic principle, Rev. Mod. Phys. 74 (2002), 825-874.

[9] D. Brill and F. Flaherty, Isolated maximal surfaces in spacetime, Commun. Math. Phys. 50 (1984), 157-165.

[10] M. Caballero, A. Romero and R. Rubio, Complete CMC spacelike surfaces with bounded hyperbolic angle in generalized Robertson-Walker spacetimes, Int. J. Geom. Methods Mod. Phys. 7 (2010), 961-978.

[11] M. Caballero, A. Romero and R. Rubio, New Calabi-Bernstein results for some elliptic nonlinear equations, Anal. Appl. 11 (2013), 1350002, 13 pp.

[12] M. Caballero, A. Romero and R. Rubio, Constant mean curvature surfaces in Generalized Robertson-Walker spacetimes, Lett. Math. Phys. 93 (2010), 85-105.

[13] Y. Choquet-Bruhat, Quelques proprits des sousvarits maximales d'une varit lorentzienne, Comptes Rend. Acad. Sci. (paris) Serie A 281 (1975), 577-580

[14] E. Calabi, Examples of Bernstein problems for some nonlinear equations, Proc. Symp. Pure Math. 15 (1970), 223-230.

[15] S.Y. Cheng and S.T. Yau, Maximal spacelike hypersurfaces in the Lorentz-Minkowski spaces, Ann. of Math. 104 (1976), 407-419.

[16] R.P. Geroch, Limits of spacetimes, Comm. Math. Phys. 13 (1969), 180-193.

[17] R.E. Grenne and H. Wu, Function theory on manifolds which possess a pole, Lect. Notes Math. 699, Springer-Verlag, New York, 1979.

[18] J. Hano and K. Nomizu, Surfaces of revolution with constant mean curvature in LorentzMinkowski space, Tohoku Math. J. 36 (1984), 427-437.

[19] T. Ishihara and F. Hara, Surfaces of revolution in the Lorentzian 3-space, J. Math. Tokushima Univ 22 (1988), 1-13.

[20] L. Karp, Subharmonic function on real and complex manifolds, Math. Z. 179 (1982), 535-554.

[21] J.L. Kazdan, Parabolicity and the Liouville property on complete Riemannian manifolds, Aspects of Math. E10, Ed. A.J. Tromba, Friedr. Vieweg and Sohn, Bonn (1987), 153-166.

[22] P. Li, Curvature and function theory on Riemannian manifolds, Surveys in Differential Geometry, Vol. II, International Press (2000), 375-432.

[23] J.E. Marsden and F.J. Tipler, Maximal hypersurfaces and foliations of constant mean curvature in General Relativity, Phys. Rep. 66 (1980), 109-139. 
[24] H. Omori, Isometric immersions of Riemannian manifolds, J. Math. Soc. Japan 19 (1967), $205-214$.

[25] B. O’Neill, Semi-Riemannian Geometry with applications to Relativity, Academic Press, 1983.

[26] B. Palmer, The Gauss map of a spacelike constant mean curvature hypersurface of Minkowski space, Comment. Math. Helv. 65 (1990), 52-57.

[27] M. Rainer and H-J. Schmidt, Inhomogeneous cosmological models with homogeneous inner hypersurface geometry, Gen. Relativity Gravitation 27 (1995), 1265-1293.

[28] A. Romero and R. Rubio, On the mean curvature of spacelike surfaces in certain 3-dimensional Robertson-Walker spacetimes and Calabi-Bernstein's type problems, Ann. Glob. Anal. Geom. 37 (2010), 21-31.

[29] A. Romero, R. Rubio and J.J. Salamanca, Uniqueness of complete maximal hypersurfaces in spatially parabolic generalized Robertson-Walker spacetimes, Classical Quantum Gravity, 30 (2013) 115007, 13 pp.

[30] R.K. Sachs and H. Wu, General Relativity for Mathematicians, Grad. Texts Math., Vol. 48, Springer-Verlag, New York, 1977.

[31] M. Sánchez, On the geometry of generalized Robertson-Walker spacetimes: curvature and Killing fields, J. Geom. Phys. 31 (1999), 1-15.

[32] A. Sommerfeld, Partial Differential Equations in Physics, Academic Press, New York, 1949.

[33] S.M. Stumbles, Hypersurfaces of constant mean curvature, Annals of Physics 133 (1981) $28-56$.

[34] A.E. Treibergs, Entire spacelike hypersurfaces of constant mean curvature in Minkowski space, Invent. Math. 66 (1982), 39-56.

[35] S.T. Yau, Harmonic functions on complete Riemannian manifolds, Commun. Pure Appl. Math. 28 (1975), 201-228.

[36] Y.L. Xin, On the Gauss image of a spacelike hypersurface with constant mean curvature in Minkowski space, Comment. Math. Helv. 66 (1991), 590-598. 